\title{
Zitterbewegung Modeling
}

\author{
David Hestenes
}

\begin{abstract}
Guidelines for constructing point particle models of the electron with zitterbewegung and other features of the Dirac theory are discussed. Such models may at least be useful approximations to the Dirac theory, but the more exciting possibility is that this approach may lead to a more fundamental reality.
\end{abstract}

\section{INTRODUCTION}

For many years I mulled over a variation of Schrödinger's zitterbewegung concept to account for some of the most perplexing features of quantum theory. I was reluctant to publish my ideas, however, because the supporting arguments were mainly qualitative, and physics tradition demands a quantitative formulation which can be subjected to experimental test. Unfortunately, the road to a quantitative version appeared to be too long and difficult to traverse without help from my colleagues. But how could I enlist their help without publishing my qualitative arguments to get their attention?

The impetus to break this impasse came from the inspring example of Asim Barut. Barut is unusual among theoretical physicists, not only for the rich variety of clever ideas he generates, but also for publishing theoretical fragments which are sometimes mutually incompatible. Though each of his papers is internally coherent, he is not afraid to contradict himself from one paper to the next or try out alternative theoretical styles. He rapidly explores one idea after another in print, searching, I suppose, for ever deeper insights. Consequently, his papers are an exceptionally rich source of new ideas, arguments, and viewpoints. It was Barut's inspiring example that convinced me finally to publish my qualitative analysis of the zitterbewegung in Ref. 1. Despite its defects, that publication did, indeed, stimulate helpful interaction with my peers and led to a mathematically wellgrounded zitterbewegung interpretation of the Dirac theory [2] which is the starting point for the present paper. In appreciation for his positive influence on these events, I dedicate this paper to Asim Barut.

The main motivation for analyzing zitterbewegung (ZBW) models is to explain the electron's spin $S$ and magnetic moment $\mu$ as generated by a local circulation of mass and charge. Experimental evidence rules out the possibility that the electron is an extended body, for the relativistic limitation on velocity implies that, to generate $S$ and $\mu$, the dimensions of the body cannot be less than a Compton wavelength $\left(\equiv 10^{-13} \mathrm{~m}\right)$, whereas scattering experiments show that the electron cannot be larger than $10^{-16} \mathrm{~m}$. This leaves open the possibility that the electron can be regarded as a point charge which generates $S$ and $\mu$ by an inherent local circular motion.

For a particle moving in a circle at the speed of light $c$, the radius of the circle $\lambda$ is related to the circular frequency $\omega$ by

$$
\lambda \omega=c=1
$$


If the particle is presumed to have mass $m$ and generate the electron spin by this motion, then

$$
|S|=\frac{1}{2} \hbar=m \omega \lambda^{2}
$$

Thus, radius (wavelength), mass, and frequency are all related by

$$
\lambda=\frac{\hbar}{2 m}=\frac{1}{\omega}
$$

This may appear to be a hopelessly simple-minded explanation for electron spin, but (surprise!!) it has been shown to be completely consistent with the mathematical structure of the Dirac theory. ${ }^{(2)}$ Thus, despite the appearance of electron mass in the Dirac equation, the electron may indeed be moving with the speed of light. Contrary to long-standing "classical" arguments, such motion can produce a gyromagnetic ratio of 2. Moreover, the complex phase factor can be interpreted as a direct representation of the circular ZBW. Thus, the phase factor

$$
e^{-i \varphi / \hbar}=e^{-i \theta / 2}
$$

is associated with the frequency $\omega=\dot{\theta}$, where differentiation is with respect to the proper time on the worldline of the center of curvature for the circular ZBW. Note that (1.4) is a half-angle representation of the circle. Comparison of (1.4) with (1.3) gives

$$
\dot{\varphi}=\frac{1}{2} \hbar \omega=m
$$

showing that $m$ must be a variable mass. It is equal to the electron rest mass $m_{0}$ only for a free particle. This interpretation of the phase $\varphi$ is derived from the standard energymomentum operator

$$
\underline{p}_{\mu}=\mathbf{i} \hbar \partial_{\mu}-e A_{\mu}
$$

Operating on the phase factor (1.4), we obtain the gauge-invariant energy-momentum vector

$$
p_{\mu}=\partial_{\mu} \varphi-e A_{\mu}
$$

Contraction with the "Dirac velocity" $v^{\mu}$ (for the center of curvature) gives

$$
p \cdot v=\dot{\varphi}-e A \cdot v
$$

For $m=p \cdot v$ this differs from (1.5), an important point to be discussed later.

The compatibility of this ZBW interpretation with the details of the Dirac theory is demonstrated in Ref. 2. Its very success, however, suggests the possibility of a deeper theory of electrons. It suggest that the Dirac theory actually describes a statistical ensemble of possible electron motions. If that is correct, it should be possible to find equations of motion for a single electron history and then derive the Dirac theory by statistical arguments. That possibility is explored in this paper, where definite equations of motion for a single point particle with ZBW are written down and discussed. With the ZBW interpretation as a guide, the equations are designed to capture the essential features of the Dirac equation.

Of course, such "working backward" from presumed statistical averages to underlying dynamics is a guessing game, prone to error in its early stages. For that reason, this paper makes no pretense of producing a definitive ZBW model. The objective, rather, is 
to motivate and describe both kinematical and dynamical aspects of the ZBW concept,

to formulate and implement basic design principles for ZBW modeling, and

to identify and sharpen issues that must be resolved to produce a fully viable theory.

This is not the place to discuss experimental tests of the ZBW.

If the resulting equations do not in fact describe a deeper reality, at least they may constitute a useful approximation to the Dirac theory, a new version of "semiclassical" approximations which have already proved their value.

\section{OBSERVABLES OF THE DIRAC THEORY}

The present paper is a continuation of work published in this journal, ${ }^{(2)}$ so some familiarity with that work can be presumed as background. In particular, spacetime algebra will be employed as the essential mathematical tool. The rudiments of spacetime algebra are explained in Ref. 2 and expanded in many references cited therein. A basic feature of the algebra is that the four $\gamma_{\mu}$ in the Dirac theory are not regarded as matrices but as vectors constituting a fixed orthonormal frame for spacetime.

As a guide for the design of a ZBW model, the formulation of Dirac observables in terms of spacetime algebra is reviewed here. The Dirac wave function for a electron can be written in the form

$$
\psi=\left(\rho e^{i \beta}\right)^{1 / 2} R
$$

where $\rho$ and $\beta$ are scalars, $i=\gamma_{0} \gamma_{1} \gamma_{2} \gamma_{3}$ is the unit pseudoscalar, and $R$ is a "unimodular" spinor, that is, an even multivector satisfying

$$
R \widetilde{R}=1
$$

The form (2.1) can be interpreted physically as a factorization of the electron wave function into a statistical factor $\left(\rho e^{i \beta}\right)^{1 / 2}$ and a kinematical factor $R$. Thus, the scalar modulus $\rho$ is interpreted as a probability density, although the interpretation of $\beta$ raises problems to be discussed later.

The kinematical factor $R=R(x)$ determines an orthonormal frame of vectors $e_{\mu}=e_{\mu}(x)$ at each spacetime point $x$ by

$$
e_{\mu}=R \gamma_{\mu} \widetilde{R}
$$

This frame describes the kinematics of electron motion. The Dirac current

$$
J=\psi \gamma_{0} \bar{\psi}=\rho e_{0}
$$

has vanishing divergence, so it determines a family of electron streamlines with tangent vector

$$
v=\frac{d x}{d \tau}=\dot{x}=e_{0}
$$

The vector field $\mathrm{v}=\mathrm{v}(\mathrm{x})$ can be interpreted statistically as a prediction of the electron velocity at each spacetime point $x$. The electron spin (or polarization) vector is defined by

$$
s=\frac{1}{2} \hbar e_{3}
$$


However, the electron spin is more properly characterized as a bivector quantity $\mathrm{S}$ defined by

$$
S=\frac{1}{2} \hbar e_{2} e_{1}=i s v
$$

The unimodularity condition (2.2) implies that the derivative of $R$ in the direction $\gamma_{\mu}$ can be written in the form

$$
\partial_{\mu} R=\frac{1}{2} \Omega_{\mu} R
$$

where $\Omega_{\mu}$ is a bivector quantity. Consequently, the derivatives of (2.3) can be written

$$
\partial_{\mu} e_{\nu}=\Omega_{\mu} \cdot e_{\nu}
$$

showing that $\Omega_{\mu}$ is to be interpreted as the rotational velocity of the frame $\left\{e_{\mu}\right\}$ when displaced in the $\gamma_{\mu}$ direction.

A physical meaning is assigned to the $\Omega_{\mu}$ by comparing them to the spin $S$. Write

$$
\Omega_{\mu} S=\Omega_{\mu} \cdot S+\Omega_{\mu} \times S+\Omega_{\mu} \wedge S=P_{\mu}+i q_{\mu}+\partial_{\mu} S
$$

where

$$
\begin{aligned}
P_{\mu} & =P \cdot \gamma_{\mu}=\Omega_{\mu} \cdot S \\
q_{\mu} & =q \cdot \gamma_{\mu}=-i\left(\Omega_{\mu} \wedge S\right)=\Omega_{\mu} \cdot(-i S)=\Omega_{\mu} \cdot(s v) \\
& =v \cdot\left(\partial_{\mu} s\right)=-s \cdot\left(\partial_{\mu} v\right) \\
\partial_{\mu} S & =\Omega_{\mu} \times S
\end{aligned}
$$

In the Dirac theory, the vector $P$ in (2.11) is implicitly interpreted as the canonical (energy) momentum, which is related to the kinetic (energy) momentum vector $p$ by

$$
P=p+e A
$$

where $A=A^{\mu} \gamma_{\mu}$ is the electromagnetic vector potential and $e$ is the electron charge. Thus, Eq. (2.11) explicitly attributes the energy-momentum of the electron to the rotation rate in the plane of the spin $S$. This is a key to the ZBW interpretation, and identifies (2.11) as the full generalization of (1.7).

Along an electron streamline, the rotational velocity is

$$
\Omega=v^{\mu} \Omega_{\mu}
$$

where $v^{\mu}=v \cdot \gamma^{\mu}$, so (2.8) yields

$$
\dot{R}=\frac{1}{2} \Omega R
$$

where the overdot indicates differentiation with respect to proper time along the streamline. The "motion" of the $e_{\mu}$, along a streamline is therefore described by

$$
\dot{e}_{\mu}=\Omega \cdot e_{\mu}
$$

In particular,

$$
\dot{v}=\Omega \cdot v
$$


becomes an equation of motion for the streamline when $\Omega=\Omega(x)$ is specifled. And $(2.13)$ yields

$$
\dot{S}=\Omega \times S
$$

which determines the spin precession along a streamline. Thus, the dynamics of electron motion can be reduced to determining $\Omega$, a matter to be discussed later.

Along a streamline, (2.10) and (2.12) yield

$$
\Omega S=\Omega \cdot S+\Omega \times S+\Omega \wedge S=v \cdot P+\dot{S}+i(\dot{s} \cdot v)
$$

This summarizes the relation of $\Omega$ to observables. Indeed, it can be solved for $\Omega$ by multiplication with $S^{-1}$.

Furthermore, (2.11) and (2.14) yield

$$
m=p \cdot v=\Omega \cdot S-e A \cdot v
$$

which defines a variable mass $m$ for the electron in accordance with the remarks in the introduction.

All the above relations are implicit in the standard Dirac theory and do not involve any approximation, so they are equally applicable to the physical interpretation of any solution of the Dirac equation. The zitterbewegung interpretation requires one further physical assumption which, however, does not alter the mathematical structure of the Dirac theory. To account for the spin $S$ as generated by electron motion, the electron must have a component of velocity in the spin plane. This is readily achieved by identifying the electron velocity with the null vector

$$
u=e_{0}-e_{2}
$$

From (2.17) it follows that, for specified $\Omega=\Omega(x)$, it has the equation of motion

$$
\dot{u}=\Omega \cdot u
$$

Its solutions are always lightlike helixes winding around the electron streamlines with velocity $v=e_{0}$.

\section{ZITTERBEWEGUNG KINEMATICS}

Now we get to the main business of this article: to construct and analyze point particle models of the electron consistent with the features of the Dirac theory described in the preceding section. The first step is to define the appropriate kinematic variables and constraints.

The electron is presumed to have a lightlike history $z=z(\tau)$ in spacetime with velocity

$$
u=\frac{d z}{d \tau}=\dot{z}, \quad \text { so } \quad u^{2}=0
$$

A proper time cannot be defined on such a curve, so an additional condition is needed below to specify the parameter $\tau$ uniquely. 
The electron motion is assumed to possess an intrinsic angular momentum, or spin, $S=S(\tau)$, a spacelike bivector with constant magnitude specified by

$$
S^{2}=-\frac{\hbar^{2}}{4}
$$

The velocity $u$ can be decomposed into a component $v$ orthogonal to $S$ and a component $\dot{r}$ in the $S$-plane defined by

$$
\begin{aligned}
& v=(u \wedge S) S^{-1} \\
& \dot{r}=(u \cdot S) S^{-1}
\end{aligned}
$$

Note that $v$ and $\dot{r}$ are merely auxiliary variables defined by these equations for purposes of analysis. This determines the decomposition

$$
u=v+\dot{r}
$$

with

$$
v \cdot \dot{r}=0
$$

and, by (3.1),

$$
v^{2}=-\dot{r}^{2}
$$

A scaling for the parameter $\tau$ is determined by assuming that

$$
v^{2}=v \cdot u=\frac{(u \wedge S)^{2}}{S^{2}}=1
$$

It will be seen later, though, that this is a mere convention, and not really a physical constraint on the model.

Equation (3.5) suggests the decomposition

$$
z(\tau)=x(\tau)+r(\tau)
$$

with

$$
v=\dot{x}
$$

This expresses the electron history $z(\tau)$ as a circulating motion with radius vector $r(\tau)$, angular momentum $S$, and a center of curvature with a timelike history $x=x(\tau)$.

All these features of our particle model correspond exactly to kinematic features of the Dirac theory. Indeed, (3.5) is the same as (2.22) with $v=e_{0}$ and $\dot{r}=-e_{2}$. It follows that the definition of a comoving frame by (2.3) and the spinor equation of motion (2.8), with their sundry implications (2.17), (2.18), (2.19), and (2.22), apply also to the particle model, the sole difference being that all quantities are defined only on the particle history $z(\tau)$ rather than as fields distributed over spacetime. As in the Dirac theory, the dynamics in those equations are entirely determined by specifying the rotational velocity $\Omega=\Omega(z(\tau))$, a matter to be discussed in the next section.

The situation is different, however, for momentum and mass. We define the momentum $p$ in a standard way by writing

$$
p=m u=m v+m \dot{r}
$$


This cannot be identified with the momentum $p$ defined in the Dirac theory by (2.11) and (2.14), because it has the rapidly fluctuating component $\mathrm{mr}$. Rather, it will be argued later that the Dirac momentum corresponds to the average of (3.11) over a ZBW period.

The mass $m$ in (3.11) remains to be defned. In accordance with the ZBW concept, we assume that it has the same origin as the spin, following (2.21) in defining it in relation to (3.11) by

$$
m=\Omega \cdot S=p \cdot v=-p \cdot \dot{r}
$$

This may differ from (2.21), however, due to averaging over a ZBW period. The essential kinematic specifications for our model are now complete, but it will be convenient to relate mass and momentum to spin by writing

$$
S=m \dot{r} \wedge r
$$

This amounts to a definition of the vector radius of curvature. Substitution into (3.12) yields

$$
\Omega \cdot(\dot{r} \wedge r)=1
$$

This generalizes Eq. (1.1), where

$$
\omega=\Omega \cdot \hat{S}=\frac{2}{\hbar} \Omega \cdot S
$$

is the ZBW (circular) frequency.

The mass $m$, the scalar radius of curvature $\lambda=|r|$, and the ZBW frequency $\omega$ all covary with changes in $\Omega$. To deduce how thay are interrelated, note that by $(2.17), \dot{r}=-e_{2}$ implies that

$$
\ddot{r}=\Omega \cdot \dot{r}
$$

which, in turn, implies

$$
\ddot{r} \cdot \dot{r}=0
$$

Also, $r^{2}=-|r|^{2}=\lambda^{2}$ implies that

$$
\dot{r} \cdot r=-\lambda \dot{\lambda}
$$

From (3.13) and (2.19), we obtain

$$
\dot{S}=\dot{m} \dot{r} \wedge r+m \ddot{r} \wedge r=\Omega \times S=m(\Omega \cdot \dot{r}) \wedge r+m \dot{r} \wedge(\Omega \cdot r)
$$

Whence,

$$
\dot{m} \dot{r} \wedge r=m \dot{r} \wedge(\Omega \cdot r)
$$

Multiplying this by $\dot{r} \wedge r$ and using

$$
(\dot{r} \wedge r)^{2}=-\frac{\hbar^{2}}{2 m^{2}}
$$

obtained from (3.2), we find

$$
\dot{m}\left(\frac{-\hbar^{2}}{4 m^{2}}\right)=m(\dot{r} \cdot r)(\Omega \cdot r) \cdot \dot{r}=m \dot{\lambda}
$$


Therefore,

$$
\frac{-\hbar^{2}}{8} \frac{\dot{m}}{m^{3}}=\frac{d}{d \tau}\left(\frac{\hbar^{2}}{4 m^{2}}\right)=\frac{d \lambda^{2}}{d \tau}
$$

Setting the integration constant to zero, we thus obtain

$$
m \lambda=\frac{1}{2} \hbar
$$

exactly as specified by (1.3). Here, however, it is clear that $m$ and $\lambda$ can be variable, with derivatives related by

$$
\frac{\dot{m}}{m}=-\frac{\dot{\lambda}}{\dot{\lambda}}
$$

It should be evident that we have a new concept of mass here, through, to a certain extent, it was already implicit in the Dirac theory. The vague concept of mass as some kind of material stuff is completely gone. No longer is vanishing mass a distinguishing feature of particles moving with the speed of light, because $p^{2}=m^{2} u^{2}=0$ here.

The relation $m=p \cdot v$ expresses the inertial property of mass as a relation between momentum and velocity. This relation conforms to the relativistic concept of mass as a measure of energy content, but that just means that mass and energy are essentially one and the same. The complementary relation $m=\Omega \cdot S=\frac{1}{2} \hbar \omega$ asserts that mass is a frequency measure. This conforms to de Broglie's original idea that the electron contains an internal clock with frequency determined by its mass, though for a free particle the ZBW frequency differs from the de Broglie frequency by a factor of 2 , and the mass varies with interactions in the present model. Moreover, the relation $m \lambda=\frac{1}{2} \hbar$ says that this frequency measures the radius of curvature of the electron worldline, so it is a thoroughly geometrical quantity. All this suggests that the mass relates our externally imposed time scale to a time scale intrinsic to the electron.

Considerations in the next section suggest that mass is a measure of self-interaction. In other words, mass measures the coupling strength of the electron to its own field. This, then, is the source of the inertial property of electron mass. Intuitively speaking, when accelerated by external fields, the electron must drag its own field along.

The direct relation of mass to ZBW frequency described here comes from assuming $m=\Omega \cdot S=\dot{\varphi}$, which differs from the relations (2.21) and (1.8) in the Dirac theory by the $e A \cdot v$ term. This difference was introduced into the particle model for a physical reason. The Dirac equation allows the value of $\dot{\varphi}$ to be changed by a gauge transformation, whereas the ZBW interpretation of $\dot{\varphi}$ as an objective property of electron motion related to its spin implies that $\dot{\varphi}$ should have a unique value. It will be argued later that the $e A \cdot v$ term may appear in the Dirac theory from time averaging which is inherent in the theory. On the other hand, if the $e A \cdot v$ term is found to be essential to account for physical facts such as the Aharonov-Bohm effect, it could be incorporated into a particle model. This is a physical issue to be resolved by further theoretical and experimental analysis.

\section{ZITTERBEWEGUNG DYNAMICS}

As already mentioned, the dynamics of electron motion is completely determined by specifying the rotational velocity $\Omega=\Omega(z(\tau))$. An explicit expression for $\Omega$ in the Dirac 
theory was derived in Ref. 3 and is discussed in Section 6. With the Dirac $\Omega$ as a guide, our problem is to guess a suitable expression for $\Omega$ in our particle model. The simplest possibility to consider is

$$
\Omega=\frac{e}{m_{0}} F+m_{0} S^{-1}
$$

where $m_{0}$ is the electron rest mass and the bivector $F$ is any specified external electromagnetic field. This determines a well-defined dynamical model which can account for a significant range of physical phenomena.

Substituting (4.1) into (2.18) and using $v \cdot S=0$, we obtain

$$
m_{0} \dot{v}=e F \cdot v
$$

which is exactly the form of the classical Lorentz force, so it is evident that classical electrodynamics can be recovered from the model. However, if (4.2) is to be regarded as an equation of motion for the center of curvature $x=x(\tau)$ with $v=\dot{x}$, it is necessary to express $F$ as a function of $x$ instead of $z$. This is most naturally done by means of the first-order Taylor expansion.

$$
F(z)=F(x+r)=F(x)+r \cdot \square F(x)
$$

where $r \cdot \square=r^{\mu} \partial_{\mu}$. Thus, the classical result will be obtained if and only if the last term in (4.3) in negligible compared to $F(x)$. Let us call this case the classical approximation. Of course, the last term in (4.3) is a ZBW effect.

Substituting (4.1) into (2.19) yields

$$
\dot{S}=\frac{e}{m_{0}} F \times S
$$

which exhibits the $g=2$ value for the gyromagnetic ratio in the classical approximation, exactly as in the Dirac theory. Note that the ZBW correction on the right side of (4.3) vanishes when $F$ is constant, so the classical approximation applies rigorously in that case, the case which has been of greatest interest in measurements of the $g$-factor.

While (4.2) determines the electron's center of curvature, and (4.4) determines the spin precession along the worldline, the remaining feature of electron motion is the ZBW frequency determined by substituting (4.1) into (3.12) or (3.15), with the result

$$
m=\frac{e}{m_{0}} F \cdot S+m_{0}
$$

Thus, the external field produces a gauge-invariant mass shift of Larmor type.

Instead of trying to integrate the equations for $\dot{v}$ and $\dot{S}$ directly, it is advisable to employ the spinor equation (2.16) which, for $\Omega$ given by (4.1), becomes with the help of (2.2) and (2.7),

$$
\dot{R}=\frac{e}{2 m_{0}} F R+\frac{2 m_{0}}{\hbar} R \gamma_{2} \gamma_{1}
$$

This equation has solutions of the form

$$
R=R_{0} e^{-\gamma_{2} \gamma_{1} \varphi / \hbar}
$$


where $R_{0}$ satisfies the "reduced equation"

$$
\dot{R}_{0}=\frac{e}{2 m_{0}} F R_{0}
$$

and the phase $\varphi$ can be obtained by integrating (4.5) with $m=\dot{\varphi}$ after $S$ has been found by integrating (4.8).

Solutions of (4.8) in the classical approximation have been found in Ref. 4 for the cases where $F$ is a constant, plane wave, or Coulomb field, and the equation $\dot{x}=v$ was integrated to find explicit expressions for the worldline in each case.

The form of (4.1) suggests various generalizations, such as

$$
\Omega=\frac{e}{m_{0}}\left(F+F_{s}\right)
$$

where $F_{s}$ is the electron's self-field, that is, the electromagnetic field of the electron itself evaluated on the electron's worldline. The self-field has the general form

$$
\frac{e}{m_{0}} F_{s}=m_{0} S^{-1}+\Omega_{e x}+\frac{e}{m_{0}} F_{R R}
$$

where $F_{R R}$ is the radiative reaction field of the electron. Since radiative reaction is not included in the Dirac theory, we can defer discussion of the $F_{R R}$ term to another occasion. The remaining terms must then describe nonradiative effects of self-interaction.

The term $m_{0} S^{-1}$ in (4.1) and (4.10), which is crucial for "generating" the electron rest mass and spin, has the bivector form of a magnetic field. This leads to the conclusion that the electron's electromagnetic selfinteraction is fundamentally of magnetic type.

This also raises the question of what form the electron's field takes at points away from the electron worldline. A major reason for developing a ZBW model in the first place was to explain the electron's magnetic dipole field. If this explanation is taken seriously, then the dipole field must be regarded as an average over a ZBW period, and the actual field must also contain a high-frequency component $F_{z b w}$ which oscillates with the ZBW frequency. As argued in Refs. 1 and 2, this has the potential for explaining some of the most prominent and perplexing features of quantum theory, such as electron diffraction and the Pauli principle, as resonant interactions mediated by the ZBW field $F_{z b w}$. Note that (4.1) can be employed to study this possibility without further modification. It is only necessary to regard the external field Fin (4.1) as including ZBW fields from external sources. However, a quantitative analysis of ZBW resonances must be deferred to another time.

Of course, the existence of a stable fluctuating ZBW field accompanying even a free electron raises questions about radiation which must be addressed eventually. The viewpoint adopted here is that the Dirac theory suggests that such fields exist, and we should push the study of their putative effects on electron motion as far as possible before attacking the fundamental problems of radiation and self-interaction. Equation (4.10) describes the coupling of the electron to its own field. Even for a free particle, the $m_{0} S^{-1}$ term implies that the electron's momentum (3.11) has a component mr which fluctuates (rotates ) with the ZBW frequency. Presumably, this reflects a fluctuation of momentum in the electron's ZBW field. The nonspecific term $\Omega_{e x}$ is included in (4.10) to cover the possibility that the 
electron's field may have nonradiating excitations which appear even in the motion of a free particle. An example will be given in the next section.

\section{ZITTERBEWGUNG AVERAGES}

It is of interest to eliminate ZBW oscillations from the equations of motion by averaging over a ZBW period for two good reasons at least. First, these high-frequency oscillations (on the order of $10^{21} \mathrm{~Hz}$ ) are irrelevant in many situations (such as the classical limit), so it is advisable to systematically eliminate the need to consider them. Second, the (energy) momentum vector in the Dirac theory does not exhibit the ZBW fluctuations of the momentum vector $p$ defined by (3.11), so it must be regarded as some kind of average $\bar{p}$ of $p$. Thus, the study of ZBW averages is essential if the Dirac theory is to be derived from an underlying ZBW substructure.

Even apart from the complication that the ZBW period $T=2 \pi / \omega$ is a variable quantity, the definition of a ZBW average is not entirely straightforward, because it must be compatible with invariant constraints on ZBW kinematics. For example, for the center-of-curvature velocity the straightforward definition of an average $\bar{v}=\bar{v}(\tau)$ by

$$
\bar{v} \equiv \frac{1}{T} \int_{0}^{\tau} v(\tau) d \tau=\frac{x(\tau)-x(0)}{T}
$$

is unsatisfactory, because it fails to preserve the constraint $v^{2}=1$. For any curved history $x(\tau)$,

$$
|x(\tau)-x(0)|>\int_{0}^{\tau}|d x|=T
$$

implies $\bar{v}^{2}>1$. This defect could be corrected by introducing a parameter $\beta_{0}$ and revising the definition (5.1) to

$$
\bar{v} \cos \beta_{0}=\frac{1}{T} \int_{0}^{\tau} v(\tau) d \tau
$$

with $v^{2}=1$ by definition. A parameter like $\beta_{0}$ does indeed appear in the Dirac theory, but we shall entertain other, probably deeper, reasons for that. Since $v$ is orthogonal to the ZBW fluctuations, it is affected by them only indirectly through the equations of motion. It appears permissible, therefore, to take $\bar{v}=v$ by definition.

To maintain the constraint $v \cdot S=0$, it is necessary to define $\bar{S}=S$ also. In Ref. 2, I made the mistake of suggesting that $v$ be defined by the ZBW average $\bar{u}$. Instead, we take $v$ as defined already by (3.3) and define $\bar{p}$ indirectly by imposing angular momentum conservation as a constraint. In Ref. 2 , it was correctly argued that compatibility with the Dirac theory requires that the average angular momentum $J$ must satisfy the decomposition into orbital and spin components defined by

$$
J=\overline{p \wedge z}=\bar{p} \wedge x+S
$$

Of course, momentum conservation on the average requires that

$$
\dot{\bar{p}}=\bar{f}
$$


where $\bar{f}$ is the average force on the particle. Angular momentum conservation then requires that

$$
\dot{J}=\dot{\bar{p}} \wedge x+\bar{p} \wedge v+\dot{S}=\bar{f} \wedge x
$$

Therefore,

$$
\dot{S}+\bar{p} \wedge v=0
$$

As noted in Ref. 3, this equation was first formulated by Wessenhoff and has been studied by many authors as a classical model for a particle with spin. Its appearance here gives it new significance as an approximation to the Dirac equation.

Defining the average mass by

$$
\bar{m}=\overline{p \cdot v}=\bar{p} \cdot v
$$

and adding it to (5.6), we obtain

$$
v \bar{p}=\bar{m}+\dot{S}
$$

This can be solved explicitly for

$$
\bar{p}=v(\bar{m}+\dot{S})=\bar{m} v+v \cdot \dot{S}=\bar{m} v+S \cdot \dot{v}
$$

where the last equality comes from differentiating $v \cdot S=0$, and it is noted that (5.6) implies as well as

$$
v \wedge \dot{S}=0
$$

as well as

$$
\bar{p} \wedge \dot{S}=0
$$

Consistency with the conservation laws thus implies that we must take (5.8) as the definition of $\bar{p}$. It will be seen in the next section that this corresponds to the Gordon decomposition in the Dirac theory. Comparison of (5.8) with (3.11) implies that

$$
\overline{m \dot{r}}=v \cdot \dot{S}
$$

an altogether reasonable result, which implies that $\bar{p}=\overline{m u}=\bar{m} v$ if and only if $v \cdot \dot{S}=0$.

The mean dynamics is determined by assuming that it is governed by the average $\bar{\Omega}$ of the rotational velocity over a ZBW period. This implies that

$$
\dot{S}=\bar{\Omega} \times S
$$

for example. Accordingly, averaging (3.12) gives

$$
\bar{m}=\bar{\Omega} \cdot S=\bar{p} \cdot v
$$

so the analog of $(2.20)$ in this case takes the form

$$
\bar{\Omega} S=\bar{m}+\dot{S}+i(\dot{s} \cdot v)
$$

Whence, comparison with (5.8) gives

$$
\bar{\Omega} S=v \bar{p}+i(\dot{s} \cdot v)
$$


The last term in (5.16) is purely kinematical, but (5.10) puts a constraint on $\dot{s}$, for the derivative of $i s=S v=S \wedge v$ is then $i \dot{s}=S \wedge \dot{v}=i(s v) \cdot \dot{v}$. Whence,

$$
\dot{s}=-(s \cdot \dot{v}) v=(\dot{s} \cdot v) v
$$

which implies

$$
\dot{s} \cdot \dot{v}=0
$$

as well as

$$
\dot{v} \cdot \dot{S}=0
$$

An equation of motion for the mass can now be derived. From the right side of (5.9), it follows that

$$
\bar{p} \cdot \dot{v}=0
$$

and (5.13) implies

$$
\bar{\Omega} \cdot \dot{S}=\bar{\Omega} \cdot(\bar{\Omega} \times S)=0
$$

for any value of $\bar{\Omega}$. Therefore, the derivative of (5.14) gives

$$
\dot{\bar{m}}=\dot{\bar{\Omega}} \cdot S=v \cdot \dot{\bar{p}}=v \cdot \bar{f}
$$

This is consistent with a general force law of the form

$$
\dot{\bar{p}}=\bar{f}=\bar{m} \bar{\Omega} \cdot v+(\dot{\bar{\Omega}} \cdot S) v
$$

However, when radiation is ignored, $\bar{f}$ should presumably depend only on the external field $F$ in (4.9). Accordingly, since (4.3) implies

$$
\overline{F(z)}=F(x)
$$

for $r=0,(5.23)$ should be replaced by

$$
\dot{\bar{p}}=\bar{f}=-\frac{\bar{m}}{m_{0}} e F \cdot v+\square\left(\frac{e}{m_{0}} S \cdot F\right)
$$

which is related to $(5.22)$ by

$$
\dot{\bar{m}}=v \cdot \bar{f}=v \cdot \square\left(\frac{e}{m_{0}} S \cdot F\right)=\frac{e}{m_{0}} S \cdot \dot{F}
$$

The force law (5.25) appears to be the most general expression for $\bar{f}$ which holds for arbitrary $F$ and is consistent with the conditions on $\bar{p}$. The mean equations of motion are thus determined by consistency conditions without a formal averaging procedure. The ratio $\bar{m} / m_{0}$ in (5.25) is strange, but a similar ratio appears in the Dirac theory. Of course, it may be that some modification of the expression for $\Omega$ in (4.9) is needed.

Substituting (5.9) into (5.25) and using (5.26) as well as (5.19), we obtain

$$
\bar{m} \dot{v}+v \cdot \cdot-S=\frac{e \bar{m}}{m_{0}} F \cdot v
$$


This equation has a nontrivial solution even for $F=0$, but it must be integrated together with Eq. (5.13) for $\dot{S}$. The general free particle solution is most easily obtained by determining the constants of motion. Of course, $\bar{p}$ is one such constant. The bivector $\bar{\Omega}$ is also constant if and only if $\dot{s}=0$, for in that case (5.16) yields

$$
\bar{\Omega}=v \bar{p} S^{-1}=i s^{-1} \bar{p}
$$

The spinor equation (2.16) then integrates to

$$
R=e^{\frac{1}{2} \bar{\Omega} \tau}
$$

so the spin undergoes constant precession described by

$$
S=R S_{0} \widetilde{R}
$$

The history $x=x(\tau)$ is most easily found by writing (5.8) in the form

$$
v=(\bar{m}+\dot{S}) / \bar{p}
$$

which integrates immediately to

$$
x(\tau)=x_{0}+\bar{m} \bar{p}^{-1} \tau+R S_{0} \bar{p}^{-1} \widetilde{R}
$$

where the fact that $\bar{p}$ commutes with $\bar{\Omega}$ has been used. This solution is a timelike helix with a precessing velocity

$$
v=R v_{0} \widetilde{R}
$$

What physical meaning can be ascribed to such a solution? Comparison with (4.10) suggests that

$$
\bar{\Omega}=m_{0} S^{-1}+\Omega_{e x}
$$

As suggested in Section 4, the $\Omega_{e x}$ may describe self-interaction due to stable excitations of the electron's ZBW field. For the "ground state self-interaction, $\Omega_{e x}=0$ which implies $\dot{S}=0$ and $\bar{m}=m_{0}$.

Returning to the general problem of ZBW averaging, we note that the net effect of the averaging is to change Eqs. (2.16) and (2.17) to

$$
\dot{R}=\frac{1}{2} \bar{\Omega} R
$$

and

$$
\dot{e}_{\mu}=\bar{\Omega} \cdot e_{\mu}
$$

This reduces the problem to determining $\bar{\Omega}$. Evidently $\bar{\Omega}$ cannot be found by simple averaging because certain consistency constraints must be satisfied. Though a thoroughly justified derivation of $\bar{\Omega}$ is not possible at this time, comparison of (5.27) with (5.36) and (4.1) suggests that it should have the form

$$
\bar{\Omega}=\frac{e}{m_{0}} F+m_{0} S^{-1}+\frac{1}{\bar{m}} \ddot{S}
$$


This differs in form from Eq. (4.1) for $\Omega$ only in the $\ddot{S}$ term. It seems, therefore, that the $\ddot{S}$ arises from the averaging, though the interpretation of (5.34) suggests that it may describe an excitation of the ZBW field and so should be included in $\Omega$ even without averaging.

Aside from (possibly) altering the form of $\Omega$, the ZBW averaging changes the functional dependence from $\Omega=\Omega(z(\tau))$ defined on the electron worldline $z(\tau)$ to $\bar{\Omega}=\bar{\Omega}(x(\tau))$ defined on the center-of-curvature worldline $x(\tau)$ This has the effect of decoupling the circular motion in the $S$-plane from the equations of motion for $v$ and $S$, so $\Omega \cdot S$ could be assigned any value without affecting $v$ and $S$. Determining the average angular velocity (or mass) of the ZBW therefore requires special considerations. Though a definitive resolution of the issue is not to be expected at this time, here is an important consideration: It may be that the difference between the ZBW mass-angle relation $m=\dot{\varphi}$ and the Dirac relation (1.8) is a consequence of averaging. This possibility can be expressed by rewriting (1.8) in the form

$$
\bar{m}=\bar{p} \cdot v=\overline{\dot{\varphi}}=\dot{\bar{\varphi}}-e A \cdot v
$$

To see how the $A \cdot v$ term might be generated by averaging, consider the line integral over a ZBW period $T=2 \pi / \omega$,

$$
\int_{0}^{T} A \cdot d z=\int_{0}^{T} A \cdot d x+\oint_{0}^{T} A \cdot d r
$$

where (3.9) has been employed to decompose it into two terms. Stokes' theorem gives

$$
\oint_{0}^{T} A \cdot d r=\int\left(d^{2} r\right) \cdot(\square \wedge A) \approx \frac{T}{h b a r} S \cdot F(x)
$$

Whence, (5.39) yields

$$
\frac{1}{T} \int_{0}^{T} A \cdot d z \approx A \cdot v+\frac{S \cdot F}{\hbar}
$$

Thus, the line integral generates the gauge-invariant average of the $S \cdot F$ "mass shift" term in (4.5), but at the expense of introducing the gauge-dependent term $A \cdot v$. Is the $A \cdot v$ term an artifact of the averaging process? If this term is really needed to account for the Aharonhov-Bohm effect, as is widely believed, then the ZBW "phase velocity" $\dot{\varphi}$ must be gauge dependent, so it does not have a unique value, which appears to be incompatible with its ZBW interpretation as a property of a unique world line. These remarks are aimed at sharpening the issue with no pretense of a final resolution.

\section{RELATION TO THE DIRAC THEORY}

The Dirac equation can be reformulated as a kind of "constitutive equation" relating the observables $\rho, v, S, p$ and $\beta$ defined in Section 2. This was done in Ref. 3, with the result

$$
\rho(\bar{p}-i q) e^{i \beta}=m_{0} \rho v-\square\left(\rho S e^{i \beta}\right)
$$

where $\square=\gamma^{\mu} \partial_{\mu}$. The symbol $p$ for the momentum vector has been replaced by $\bar{p}$ here, because, as in Section 5, we wish to consider $\bar{p}$ as the average of a particle momentum 
$p$. Only, here the average is conceived as taken over a statistical ensemble of possible momenta as well as over a ZBW period. So $\bar{p}=\bar{p}(x)$ is a vector field, and $\rho \bar{p}=\rho(x) \bar{p}(x)$ is a momentum density, just as the Dirac current $\rho v=\rho(x) v(x)$ is a particle density. The vector part of (6.1) gives the so-called "Gordon decomposition" of the Dirac current

$$
m_{0} \rho v=\rho k+\square \cdot\left(\rho S e^{i \beta}\right)
$$

where the "Gordon current"

$$
\rho k=\rho(\bar{p} \cos \beta-q \sin \beta)
$$

is interpreted as a "conduction current" and $\rho S e^{i \beta}$ is said to be a "spin density." But this interpretation is confounded by the peculiar role of $\beta$. In particular, it seems inconsistent with the identification of $\rho S$ as spin density, which comes from angular momentum conservation.

A more sensible interpretation comes from rewriting (6.1) in the form

$$
\rho(\bar{p}-i q)=m_{0} \rho v e^{-i \beta}-\square(\rho S)+i(\square \beta) \rho S
$$

Its vector part,

$$
\rho \bar{p}=\rho v m_{0} \cos \beta-\square \cdot(\rho S)+\rho(i S) \cdot \square \beta
$$

separates the momentum density $\rho \bar{p}$ into a part which depends on the spin density $\rho S$ and a part which does not. This equation should be compared with Eq. (5.9) for the time-average momentum. The difference between the two equations is presumably due to ensemble averaging, so (5.9) should be recoverable by some kind of approximation to (6.5). Toward that end, we neglect the derivative of $\rho$ in

$$
\bar{p}=v m_{0} \cos \beta-\square \cdot S+(v s) \cdot \square \beta
$$

Next, to decouple Dirac streamlines the spatial variation between them must be ignored; in other words, assume that the variation of all quantities in (6.6) is along streamlines. This "streamline approximation" can be expressed formally by writing

$$
\square=v \frac{d}{d \tau}
$$

in $(6.6)$, so it becomes

$$
\bar{p}=v m_{0} \cos \beta-v \cdot \dot{S}-s \dot{\beta}
$$

Comparing this with (5.9), the first thing to note is that the sign corresponding $v \cdot \dot{S}$ term is opposite. This discrepancy could be eliminated by changing a sign in the initial equation (5.3) determining the relation between $\bar{p}$ and $S$. However, Eq. (5.6), from which (5.9) is derived, is also derivable from the Dirac theory. Thus, the discrepancy is perplexing, but, with full confidence in the internal consistency of the Dirac theory, let us presume that it can be resolved and move on.

Comparison of (6.8) with (5.9) or (5.7) suggests

$$
\bar{m}=\bar{p} \cdot v=m_{0} \cos \beta
$$


In other words, the invariant $\cos \beta$ describes a variation in mass. This interpretation is strongly supported by a virial theorem derived from the Dirac equation in Ref. 5, which proves that the entire energy shift of an electron in a Coulomb field can be ascribed to $\cos \beta$.

The last term in (6.8) does not appear in (5.9), but it may indicate a need to generalize the derivation of (5.9). It follows from (6.8) that

$$
\dot{\beta}=-\bar{p} \cdot s^{-1}
$$

which could play a complementary role to (6.9).

By strict analogy with the reasoning that led to (5.12), to obtain (6.5) by averaging over ZBW oscillations, we should presumably have

$$
\overline{m \dot{r}}=-\square \cdot(\rho S)+\rho(i S) \cdot \square \beta
$$

where the overbar now denotes an ensemble average as well as the time average in (5.12).

To complete our discussion of the Dirac equation (6.4), we will need to consider its trivector part

$$
\rho q=-\rho v m_{0} \sin \beta+\square \cdot(\rho S i)+\rho S \cdot \square \beta
$$

One can obtain from this

$$
\rho q \cdot s=s^{2} \square \cdot(\rho v)-\rho s(s \cdot \square v)
$$

and

$$
\rho q \cdot v=-\rho m_{0} \sin \beta+\rho v \cdot \dot{s}-\square \cdot(\rho s)
$$

Using the definition of $q$ by (2.12) to evaluate the left side, these equations yield

$$
\square \cdot(\rho v)=0
$$

and

$$
\square \cdot(\rho s)=-\rho m_{0} \sin \beta
$$

The first of these is the well-known conservation law for the Dirac current, and in the approximation (6.7) it reduces to the identify $v \cdot \dot{v}=0$. The physical significance of $(6.14 \mathrm{~b})$ is difficult to divine. In the approximation (6.7) it reduces to

$$
v \cdot \dot{s}=-m_{0} \sin \beta
$$

which shouid be considered for a particle model along with (6.9) and (6.10). The main issue is whether $\beta$ is an artifact of the averaging process or actually describes a real feature of the particle (such as variable mass). Of course, it could be a combination of the two.

Besides (6.13a,b), Eq. (6.12) implies two other relations which can be found by evaluating $q \cdot S$. However, the result is contained in the dynamical relation which we consider next.

The dynamics of electron motion along a streamline in the Dirac theory is completely determinied by the rotational velocity $\bar{\Omega}$, which can be calculated from the Dirac wave function. An explicit expression for $\bar{\Omega}$ in terms of observables was found in Ref. 3 to have the form

$$
m_{0} \bar{\Omega}=e F e^{i \beta}+Q+\left(m_{0} \cos \beta+e v \cdot A\right) S^{-1}
$$


where $F$ is the external electromagnetic field and $Q$ is a complicated function of $\rho, \beta$, and $S$ which need not be exhibited here. The overbar is to remind us that $\bar{\Omega}$ is to be regarded as an average in the sense specified above.

Inserting (6.16) into (2.18) and (2.19), we get equations of motion for the velocity and the spin precession along a streamline:

$$
\begin{gathered}
m_{0} \dot{v}=\left(e F e^{i \beta}+Q\right) \cdot v \\
m_{0} \dot{S}=\left(e F e^{i \beta}+Q\right) \times S
\end{gathered}
$$

These are exact equations in the Dirac theory. Their most surprising feature is the $\beta$ dependent "duality rotation" of the external field $F$. This kind of rotation mixes electric and magnetic fields, so it may well be detectable experimentally, but there has been no effort to do so thus far. There seems to be nothing in the ZBW process to produce such an effect, which again suggests that the peculiar parameter $\beta$ arises from the process of averaging.

The term $Q \cdot v$ generalizes what Bohm has called the "quantum force." It determines the "bunching up" and "thinning out" of streamlines at maxima and minima in diffraction patterns. If the speculations about ZBW fields in Section 4 are on track, this term must describe statistical features of electron motion due to ZBW interactions. It may also include self-interaction. Indeed, in the approximation (6.7) with $\beta=0$,

$$
Q=-\ddot{S}
$$

in agreement with (5.37), except for the sign. The consequences of this approximation have been discussed at length in Ref. 5 , with results essentially in agreement with those in Section 5 .

\section{REFERENCES}

1. D. Hestenes, "Quantum mechanics from self- interaction," Found. Phys. 15, 63-87 (1985).

2. D. Hestenes, "The Zitterbewegung interpretation of quantum mechanics," Found. Phys. 20, 1213-1232 (1990). Further references are contained therein.

3. D. Hestenes, "Local observables in the Dirac theory," J. Math. Phys. 14, 893-905 (1973).

4. D. Hestenes, "Proper dynamics of a rigid point particle," J. Math. Phys. 15, 1778-1786 (1974).

5. R. Gurtler and D. Hestenes, "Consistency in the formulation of the Dirac, Pauli, and Schroedinger theories," J. Math. Phys. 16, 573-583 (1975).

6. D. Hestenes, "On decoupling probability from kinematics in quantum mechanics," in Maximum Entropy and Bayesian Methods (Dartmouth College, 1990) (Kluwer, Dordrecht, 1991), pp. 161-183. 